\title{
3D Localization Technique with Mobile Robot for Improving Operability of Remote-Control Devices
}

\author{
Masaya Yoshida, Kiyohito Yoshihara \\ KDDI R\&D Laboratories, Inc. \\ Green and M2M Application Laboratory \\ Iidabashi, Japan \\ Email: my-yoshida, yosshy@kddilabs.jp
}

\author{
Amarlingam M, Vinod Kumar Netad, P Rajalakshmi \\ Department of Electrical Engineering \\ Indian Institute of Technology Hyderabad \\ Hyderabad, India \\ Email: ee13p1003, ee13m1033, raji@iith.ac.in
}

\begin{abstract}
Electrical devices which are controlled remotely by a smartphone have recently been spreading. With some typical existing systems, a user needs to identify a target device to control with only its less user-friendly ID. A system for association between actual position of the device and its position on smartphone display would allow the user to identify it easily. We call such a system "Smart Interaction System". One of the key technologies for Smart Interaction System is localization of the device. Localization technique using some anchors with the given position to measure received signal strength indication (RSSI) of a device have been developed. They incur user burden to deploy the anchors and measure their positions. Thus alleviating the burden is still a technical challenge. As a solution to the challenge, this paper proposes a new 3D localization technique with a mobile robot including a floor cleaning robot. The mobile robot would reduce the burden by measuring RSSI while cleaning floor instead of the anchors. The proposed localization technique has been implemented with a floor cleaning robot. Experimental results show that the proposed localization technique provides the position of the device with approximately $1000 \mathrm{~mm}$ of estimation error and is useful for Smart Interaction System.
\end{abstract}

Keywords-Localization; RSSI; 3D; Mobile Robot

\section{INTRODUCTION}

The latest electrical devices including a light and an air conditioning have a wireless communication module and a smartphone can be used as a controller of the devices. Fig. 1 shows an example where a user controls the lights and the air conditioning remotely with a typical existing system. In this example, the list of the device IDs is shown on the smartphone display. The user selects a device to control from the list. If the user does not know which device corresponds to ID:XXX in advance, he cannot tell which device will be actuated when he clicks the device of ID:XXX on the smartphone display. On the other hands, Fig.2 shows an example of a system using positions of the devices. In this system, the icon for the device is displayed at the position on the smartphone display associated with actual position of the device. This graphical indication would allow the user to identify the device to control easily. For instance, the system makes it possible to actuate the device in the right side of the user when the user controls the right icon on display. We call such a system "Smart Interaction System". To develop Smart Interaction System, the spatial information of devices is required. Assuming a dozen of the remote-control devices, from 10 to 20 devices, are installed

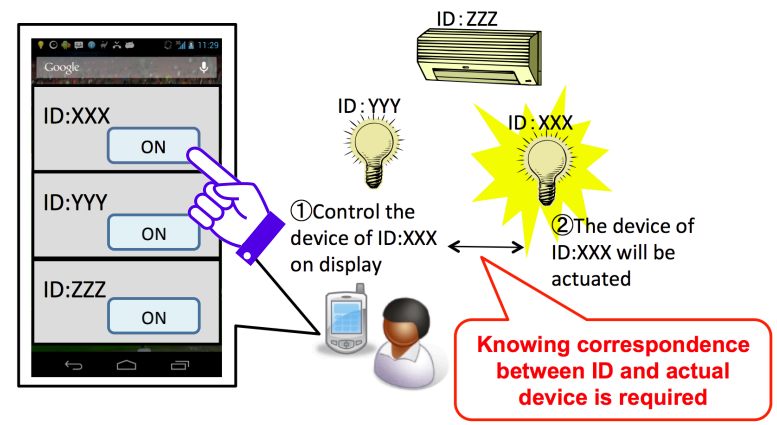

Fig. 1: Typical existing system

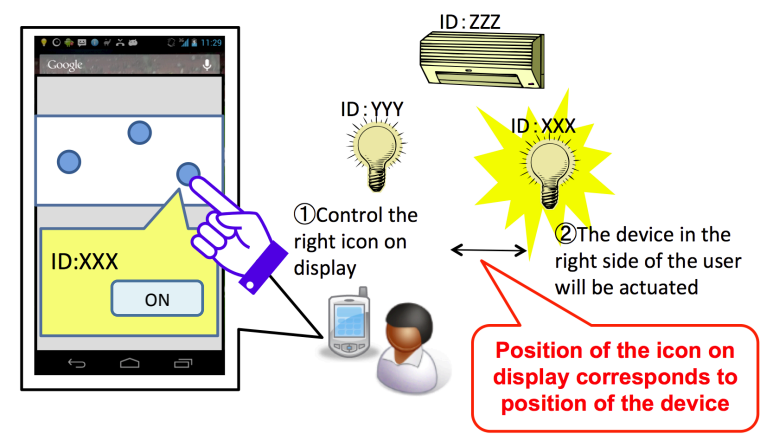

Fig. 2: Smart Interaction System

in each house in the near future, it is hard or painstaking to manually measure the position of all the devices and input them to the system. Therefore Smart Interaction System with automatic semantic labeling would be more practical to improve operability of the remote-control devices.

Achieving Smart Interaction System will need the development of localization technique of the device. Localization techniques based on received signal strength indication (RSSI) have been developed [1]-[11]. These existing localization techniques require some anchors with the given position to measure RSSI. They still incur user burden to deploy the anchors and measure their positions. Thus alleviating the burden is still a technical challenge. As a solution to the challenge, we propose a new 3D localization technique with a mobile robot such as a floor cleaning robot. The mobile robot would reduce the burden by measuring RSSI while cleaning floor instead of the anchors. By using the wide-spreading cleaning robot, the measurement of RSSI can be piggybacked on cleaning 
chores. The entire system including the proposed localization technique and Smart Interaction System has been implemented with a mobile cleaning robot. We performed experiments in indoor environment and evaluated estimation error of the localization technique.

The rest of the paper will be organized as follows. Section II shows related work on localization of a device. Section III details the proposed 3D localization technique with a mobile robot. Section IV mentions the implementation of the proposed localization and Smart Interaction System. Section V describes the experiments with the implemented system and analysis of the results. Finally, section VI concludes the paper.

\section{RELATED WORK}

In the existing localization techniques based on RSSI, some anchors are used to measure the RSSI of the devices. The position of a device is estimated by computing the distance between the device and the anchor from measured RSSI. It is well known that the radio wave on wireless communication propagates progressively with distance from a transmitter. The distance is estimated from the propagation model. The papers $[1,2]$ give a common propagation model as:

$$
p=A-10 N \cdot \log _{10}\left(\frac{d}{d_{0}}\right)
$$

where $p$ is the mean received power in $\mathrm{dB}$ at the distance $d, A$ is the received power in $\mathrm{dB}$ at the reference distance $d_{0}$ and $N$ is the path loss exponent. The localization techniques which computes the distance from the propagation model have been proposed in [3-5].

The distance derived from path loss is error-prone due to fading and multipath effect in real environment leading to inaccurate localization. In particular, in indoor environment, wall and ceiling might reflect the radio wave and make a large error due to gap between ideal and reality. To overcome the gap, localization techniques based on path loss model of indoor environment has been proposed in [6]. The authors of [7] performed an experiment of RSSI collection in a building and formulate propagation model in indoor environment. The paper [8] models radio propagation of wireless communication module based on IEEE 802.11n indoors. In [9], a propagation model mitigating the impacts caused by multipath effect is proposed to improve the performance of the maximum likelihood localization technique.

Most of the localization techniques focus on estimating 2D position of a device. While some applications require only the 2D position, others should consider the height of a device as well as its 2D position. The paper [10] provides survey on 3D localization technique of sensor nodes. The authors of [11] extend and implement one of famous techniques named MinMax algorithm for 3D localization. Smart Interaction System also needs 3D position to specify the devices at the different heights.

In many 2D and 3D RSSI-based localization techniques including [1]-[11], some anchors whose position is prerequisite must be used to collect RSSI. A user needs to deploy the anchors and manually measure their positions. Moreover, manual RSSI measurement is also prerequisite to determine the channel parameters ( $A$ and $N$ in equation (1)) for each

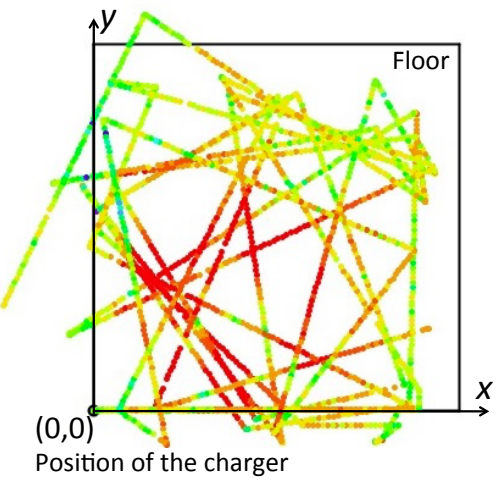

Fig. 3: An example of collected RSSI by a mobile robot

environment. We propose a 3D localization with no need of such a laborious work in the next section.

\section{PRoposed 3D LOCALIZATION WITH A MOBILE ROBOT}

The proposed technique consists of the following 2 steps.

- $\quad$ Step A: RSSI collection with a mobile robot

- $\quad$ Step B: 3D Localization with collected RSSI

1) $2 \mathrm{D}$ localization

2) Height estimation

In Step A, a mobile robot collects RSSI of the devices. The mobile robot moves around autonomously while calculating its own position. That would cut out the need of deployment of the anchors and measurement of their positions. We assume that a floor cleaning robot is used as the mobile robot. By using the wide-spreading floor cleaning robot, the RSSI collection can be piggybacked on cleaning chores.

In Step B, the positions of devices are estimated from RSSI collected in Step A. In addition to the 2D localization like many of existing techniques, we estimate the height of the devices to distinguish the devices placed at the different heights in Smart Interaction System. The proposed 3D localization technique works without the channel parameters which are required in the existing localization techniques. These steps will be detailed below.

\section{A. RSSI collection with a mobile robot}

We assume that a mobile robot has wheels and moves on the floor of area where the devices are deployed. The robot measures RSSI of each device repeatedly while cleaning. After finish cleaning, the robot will be connected to a charger which is fixed on the floor. The position of the charger is defined as a reference point $(0,0)$. X-axis is determined with the direction of the wheels when the robot is connected to the charger and Y-axis is at 90 degree angle of the wheels, respectively. While the robot measures RSSI, the relative position of the robot $(x, y)$ from the reference point is calculated by odometry. The odometry is a simple technique to determine the relative position of a robot as explained in [12]. The relative position of robot is calculated from the number of rotations of the robot's wheels. An example of RSSI collected by the mobile robot is shown in Fig. 3. A red point represents higher RSSI while green one represents lower RSSI (in black and white printing, 


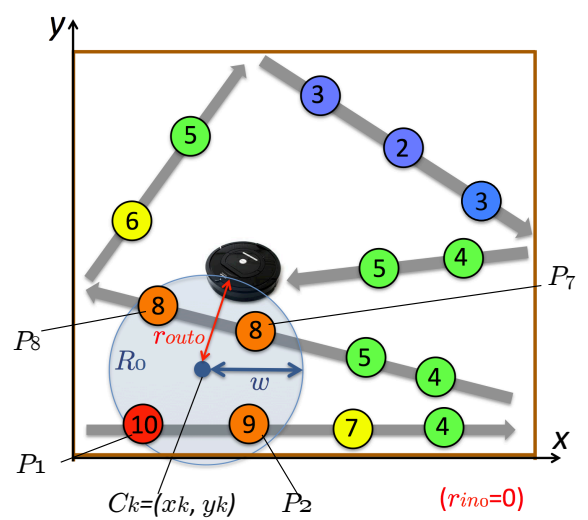

Fig. 4: Example 1: $R_{0}$ with $r_{i n 0}, r_{\text {out } 0}$

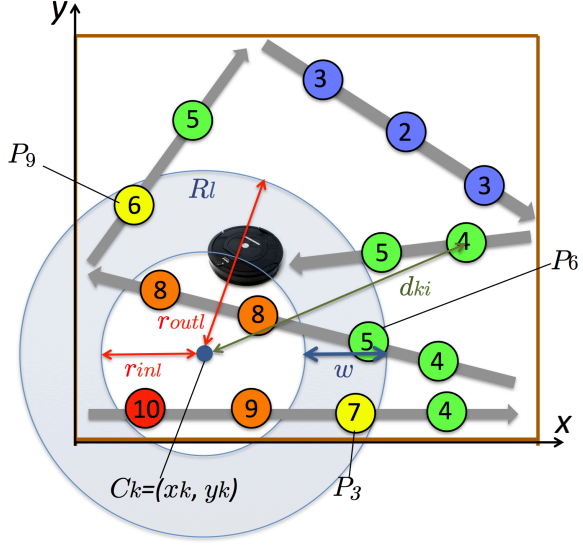

Fig. 5: Example 2: $R_{l}$ with $r_{i n l}, r_{\text {outl }}$

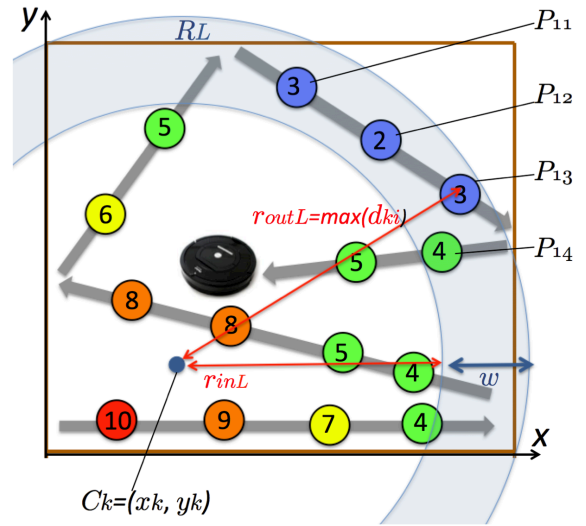

Fig. 6: Example 3: $R_{L}$ with $r_{i n L}, r_{\text {out } L}$ a darker point represents higher RSSI while a brighter one represents lower RSSI).

\section{B. 3D Localization with collected RSSI}

For 2D localization, we harness the characteristic of omnidirectional antenna that the radio wave is emitted concentrically in a horizontal direction. For height estimation, we use the maximum likelihood algorithm with no prior knowledge of the propagation model parameters.

1) 2 D localization: An omnidirectional antenna emits the radio wave concentrically in a horizontal direction. Thus, RSSI measured at the same distance from a transmitter should be about the same value like a circular pattern. Conversely, the center of the circular pattern of RSSI is likely to be the 2D position of the device. The main idea of the proposed 2D localization technique is to find the center of the circular pattern of RSSI. To find the center of the circular pattern of RSSI, first the defined area is divided into some circular rings centered at an arbitrary point. Next, the variance of RSSI is computed over each ring. Finally, the point which minimizes the mean of the variances will be regarded as the center of the circle, which is the 2D position of the device. Specifically, the proposed technique estimates the $2 \mathrm{D}$ position of the device $X_{e}=\left(x_{e}, y_{e}\right)$ as follows. Figs. 4-6 show examples how the proposed technique works. The points in Figs. 4-6 represent the positions where the mobile robot measures RSSI. The number in each point represents RSSI. RSSI measured at the position $P_{j}=\left(x_{j}, y_{j}\right)$ is denoted by $r s s i_{j}$.

For an arbitrary point $C_{k}=\left(x_{k}, y_{k}\right)$ in the defined area, $R_{l}$ denotes $l^{t h}$ ring centered at $C_{k} . r_{i n l}$ denotes the radius of the inside circle of the ring $R_{l}$ and $r_{\text {outl }}$ denotes the radius of the outside circle of the ring $R_{l}$. The width of each ring is a constant parameter $w$. We set $r_{i n 0}=0$ and $r_{o u t 0}=w$, then $R_{0}$ is a circle of radius $w$ (Fig. 4). $r_{i n l+1}$ and $r_{\text {outl }+1}$ are increased as below.

$$
\begin{aligned}
r_{i n l+1} & =r_{i n l}+w, \\
r_{\text {outl }+1} & =r_{\text {outl }}+w .
\end{aligned}
$$

The set of points $P_{j}$ in the ring $R_{l}$ is denoted by $A_{l}=\left\{P_{j} \mid P_{j}\right.$ is located in $\left.R_{k l}\right\}$. For example, $A_{0}=\left\{P_{1}, P_{2}, P_{7}, P_{8}\right\}$ in Fig. 4.
$E_{l}$ denotes the mean of RSSI values in ring $R_{l}$ and can be calculated by equation (4).

$$
E_{l}=\frac{\sum_{P_{j} \in A_{l}} r s s i_{j}}{n_{l}}
$$

where $n_{l}$ is the number of the points $P_{j}$ in the ring of $R_{l}$. We also calculate the variance of RSSI values $V_{l}$ by equation (5).

$$
V_{l}=\frac{\sum_{P_{j} \in A_{l}}\left(r s s i_{j}-E_{l}\right)^{2}}{n_{l}} .
$$

For instance, in the case shown in Fig. 5, $V_{l}$ is calculated from $r s s i_{3}=7, r s s i_{6}=5$ and $r s s i_{9}=6 . V_{l}$ is repeatedly computed while $r_{\text {out } L} \leq \max _{j}\left(d_{k j}\right)$. $L$ represents the index for the ring which contains the point with largest distance from $C_{k}$ (Fig. 6). $d_{k j}$ denotes the distance between $C_{k}$ and $P_{j}$. Equation (6) is used to find the mean of the variance in the rings centered at $C_{k}$.

$$
E V_{k}=\frac{\sum_{l}\left(V_{k l}\right)}{m_{k}}
$$

where $m_{k}$ is the number of the rings for $C_{k}$. Finally the estimated position $X_{e}$ is determined by the equation (7).

$$
X_{e}=C_{k} \text { which minimizes } E V_{k} \text {. }
$$

2) Height estimation: After estimating the 2D position of the device, the proposed localization technique estimates the height of the device with the estimated 2D position.

The proposed localization technique is based on the maximum likelihood localization technique. As discussed in the section II, the existing localization techniques require the preliminary experiment to determine the channel parameters. To eliminate the need of the preliminary experiment, in the proposed localization technique, the channel parameters are considered as the unknown variable as well as the height of the device. Then likelihood of received RSSI for each potential height and the potential channel parameters are computed. The height which maximizes the likelihood is selected as the estimated height.

Specifically, the proposed technique estimates the height $z_{e}$ of the device as follows. First one postulated height $z^{\prime}$ is 


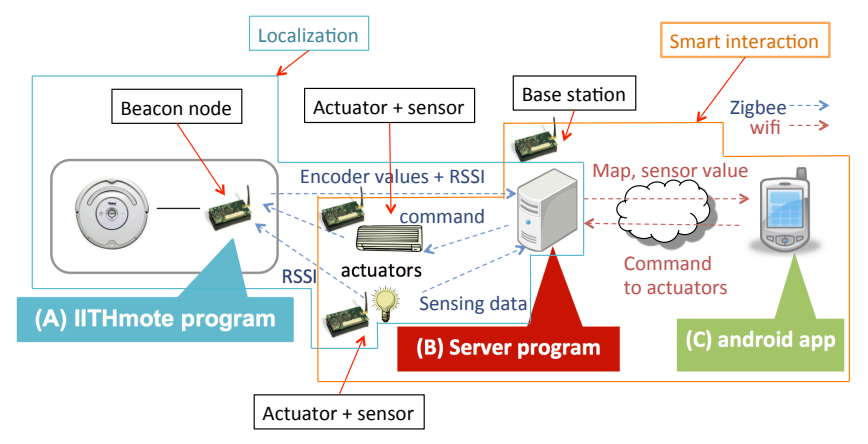

Fig. 7: Two parts: Localization and Smart Interaction

given and then the postulated RSSI $p_{j}$ for each point $P_{j}$ is calculated from equation (8).

$$
p_{j}=A^{\prime}-10 N^{\prime} \cdot \log _{10}\left(\frac{d_{j}}{d_{0}^{\prime}}\right) .
$$

$d_{j}$ is the distance between the postulated position $\left(x_{e}, y_{e}, z^{\prime}\right)$ and the point $P_{j}$. $A^{\prime}$ is mean received power at the reference distance $d_{0}^{\prime} . N^{\prime}$ is the postulated channel parameter. We consider the postulated height $z^{\prime}$ as the reference distance $\left(d_{0}^{\prime}=z^{\prime}\right)$ and compute $A^{\prime}$ by the equation (9).

$$
A^{\prime}=\frac{\sum_{P_{j} \in A_{0}} r s s i_{j}}{n_{0}} .
$$

In reference to [1], the likelihood $L$ that the postulated height $z^{\prime}$ and postulated channel parameter $N^{\prime}$ are correct is represented as:

$$
L=\prod_{j}\left\{\exp \left[-\frac{1}{2}\left(\frac{p_{j}-r s s i_{j}}{\sigma_{d B}}\right)^{2}\right]\right\}
$$

where $\sigma_{d B}$ is the standard deviation of RSSI. Here we give $\sigma_{d B}=\sqrt{V_{0}}\left(V_{0}\right.$ is computed in $2 \mathrm{D}$ localization). From the above, the likelihood $L$ is a fuction of $z^{\prime}$ and $N^{\prime}$, thus it can be represented by the equation (11).

$$
L=f\left(z^{\prime}, N^{\prime}\right)
$$

Finally the estimated height $z_{e}$ is determined as shown in the following equation (12).

$$
z_{e}=\underset{z^{\prime}}{\arg \max }\left(f\left(z^{\prime}, N^{\prime}\right)\right) .
$$

\section{IMPLEMENTATION}

Implementation of the system based on proposed localization technique consists of two parts, one is localization and another one is Smart Interaction which is an application to control the devices. The clear division of these two parts is shown in Fig. 7.

\section{A. Localization}

In our system implementation, instead of using anchors a moving robot, Roomba is used. Roomba is a cleaning robot produced by iRobot corporation. It provides a user interface to program it easily and specification of the interface is available on [13]. An anchor called beacon node is interfaced with Roomba through serial port. The beacon node controls the Roomba by sending commands through serial port. As the

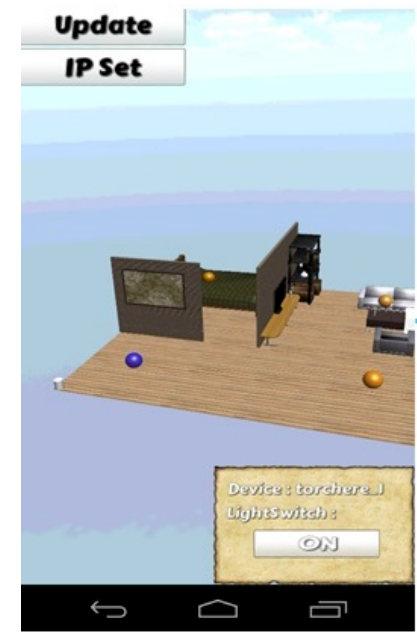

Fig. 8: 3D GUI of Smart Interaction application

beacon node is placed on Roomba, Roomba's mobility will be considered as beacon node mobility.

To determine the position of Roomba, we implemented odometry as [12] and validated the accuracy of odometry was enough for our application. The beacon node will have RSSI with respect to target nodes through wireless communication with a period of 250 milliseconds while Roomba is moving in random way in side defined area and calculate RSSI values from target nodes. After this beacon node will form a location packet (a packet which contains location details of Roomba and respective RSSI value from target nodes) and send it to a server. The server computes the position of the devices based on the proposed localization algorithm explained in section III-B.

We use IITH Motes as the beacon node and the target node. IITH Mote [14] is in-house developed ZigBee IEEE standard 802.15.4 compliant wireless communication modules.

\section{B. Smart Interaction}

The second part of the system is Smart Interaction. The Smart Interaction application is developed with Unity and runs on android platform having internet connectivity.

The application has a 3D GUI like a inside room as shown in Fig.8. A square floor, walls and some furniture are placed on fixed locations. The application is programmed to control and access all the electrical devices inside a room or building, thus being able to indicate their current state like 'on' or 'off' through server. This information can be viewed through the application GUI by clicking update button.

To access to the server from the application, a user can input IP address of the server by clicking IP Set button. A 3D map of devices locations inside room which is a result of the implemented system will be shown on the 3D GUI. The location of the charger $(0,0,0)$ is set at a corner of the floor in 3D space, and the location of devices is shown as small sphere. When a user clicks on the sphere, it will show the respective electrical device information in terms of its current state like 'on' or 'off'. With this, a user can identify a specific device to control. 


\section{EXPERIMENT AND ANALYSIS}

\section{A. Experimental settings}

To analyze the estimation error of the proposed localization technique and evaluate the operability of Smart Interaction System, we conduct experiments with the implemented system.

In this paper, we assume the situation where from 10 to 20 devices are installed in a house. To cover the situation, we placed total 45 devices in a room over the experiments. The experiments are performed in a room by considering an area of $7000 \mathrm{~mm} * 6500 \mathrm{~mm}$ and height $2500 \mathrm{~mm}$. The size of the room can be regarded as an average room size. For each experiment, 9 target nodes are arranged at 9 locations at intervals of $2000 \mathrm{~mm},(1800,1370, H) \mathrm{mm},(3800,1370$, $H) \mathrm{mm},(5800,1370, H) \mathrm{mm},(1800,3370, H) \mathrm{mm}$, (3800, $3370, H) \mathrm{mm},(5800,3370, H) \mathrm{mm},(1800,5370, H) \mathrm{mm}$, $(3800,5370, H) \mathrm{mm}$ and $(3000,5370, H) \mathrm{mm}$. All the target nodes $(\mathrm{X}, \mathrm{Y})$ co-ordinates are fixed, $H$ indicates height of the target nodes.

Total 25 experiments are performed for 5 different height and 5 different transmit power. The target nodes are placed at height $H=500 \mathrm{~mm}, 1000 \mathrm{~mm}, 1500 \mathrm{~mm}, 2000 \mathrm{~mm}$ and $2500 \mathrm{~mm}$ for 3D localization. Transmit power $P_{t x}$ is set to $3 \mathrm{dBm}$, $1.6 \mathrm{dBm},-0.2 \mathrm{dBm},-3.2 \mathrm{dBm}$ and $-7.2 \mathrm{dBm}$ for the target nodes. For the beacon node, transmit power is set to $3 \mathrm{dBm}$.

Each experiment consists of 20 trials. Duration of each trial is 2 minutes. In each trial, Roomba with the beacon node moves randomly in the defined experimental area and collects RSSI values from the target nodes.

We analyze 3D estimation error and 2D estimation error of the proposed localization technique. The 3D estimation error $\operatorname{err}_{3 D}$ and 2D estimation error $\operatorname{err}_{2 D}$ are calculated by equations 13 and 14, respectively.

$$
\begin{gathered}
\operatorname{err}_{3 D}=\sqrt{\left(x_{e}-x_{a}\right)^{2}+\left(y_{e}-y_{a}\right)^{2}+\left(z_{e}-z_{a}\right)^{2}}, \\
\operatorname{err}_{2 D}=\sqrt{\left(x_{e}-x_{a}\right)^{2}+\left(y_{e}-y_{a}\right)^{2}} .
\end{gathered}
$$

$\left(x_{e}, y_{e}, z_{e}\right)$ represents the estimated coordinate of the target node. $\left(x_{a}, y_{a}, z_{a}\right)$ represents the actual coordinate of the target node. For comparison, we also analyze the estimation error of Min-Max [11]. For both proposed protocol and Min-Max, the same input of RSSI are used to estimate the positions of the devices. Although Min-Max can be used for 3D localization, it requires RSSI collection in $3 \mathrm{D}$ space. Here we analyze only 2D estimation error for Min-Max because the Roomba moves only on the floor (2D space) to collect RSSI.

In the proposed localization, we used $w=100(\mathrm{~mm})$ to find the positions of the devices. In Min-Max, $A=-61.8(\mathrm{dBm})$ and $n=1.89$, which are derived from prior experiments, are used in equation (1).

\section{B. Experimental results analysis}

Fig. 9 shows the mean estimation error of proposed localization technique and Min-Max. The 3D and 2D estimation error of both the proposed localization technique and Min-Max decrease as the number of trials. The proposed localization technique gives the smaller $2 \mathrm{D}$ estimation error compared to Min-Max after 6 trials (12 minutes) are performed. According

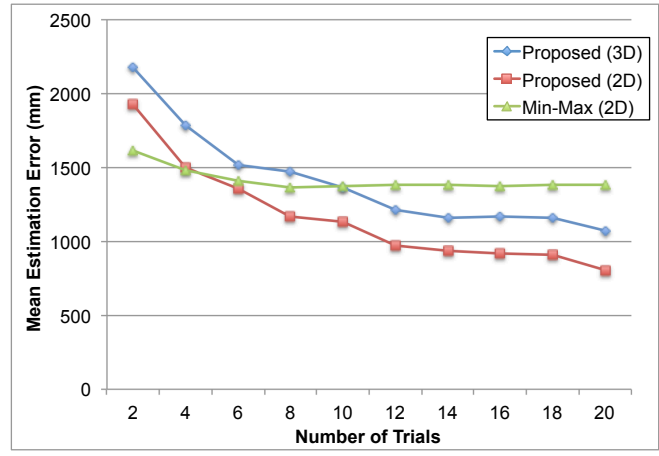

Fig. 9: Number of trials vs. mean estimation error

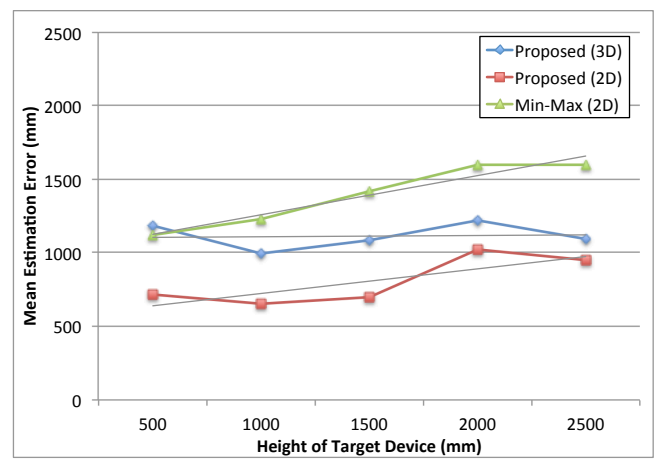

Fig. 10: Height of target device vs. mean estimation error

to [11], 3D estimation error of Min-Max in similar experimental setting is approximately $2 \mathrm{~m}$, which is higher than one of the proposed localization technique.

Fig. 10 shows that the mean estimation error for the different heights of the target nodes. The graph shows the trend that the mean estimation error increases as the height of the device. This can be caused by the longer distance between the node at higher place and the Roomba on the floor. The radio wave will be exposed through the long distance and affected by various factors such as a multipath fading.

Fig. 11 shows the mean estimation error for the different transmit power. From the result, the estimation error of the proposed localization technique is less affected by the transmit power. Although the transmit power of all devices might not be always the same if a variety of remote-control devices are installed in house, the proposed localization technique can be useful for estimating the device positions.

Fig. 12 shows the mean estimation error for $x$-axis, $y-$ axis and z-axis in 25 experiments. From the graph, the mean estimation errors for $\mathrm{x}$-axis, $\mathrm{y}$-axis and $\mathrm{z}$-axis are smaller than $1000 \mathrm{~mm}$.

\section{Discussion}

In the experiments, we assume an average room size and place the devices at intervals of $2000 \mathrm{~mm}$. In this situation, as long as the estimation error is smaller than $1000 \mathrm{~mm}$ for each axis, the icons of the devices in Smart Interaction System will not be interchanged left and right on the smartphone display. This means that a user can identify a device to control by finding the position of the icon on the display corresponding 


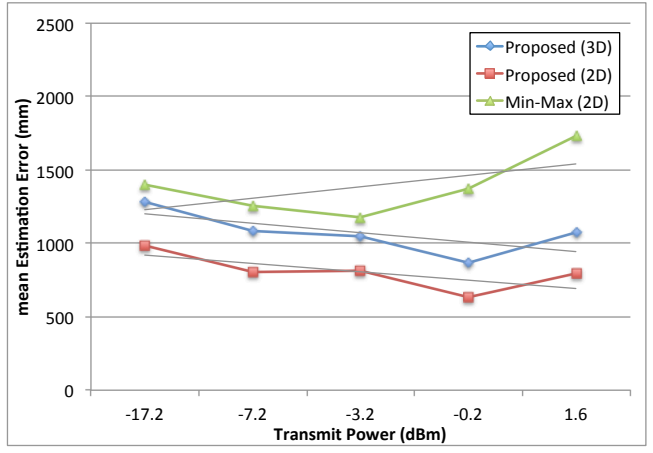

Fig. 11: Transmit power vs. mean estimation error

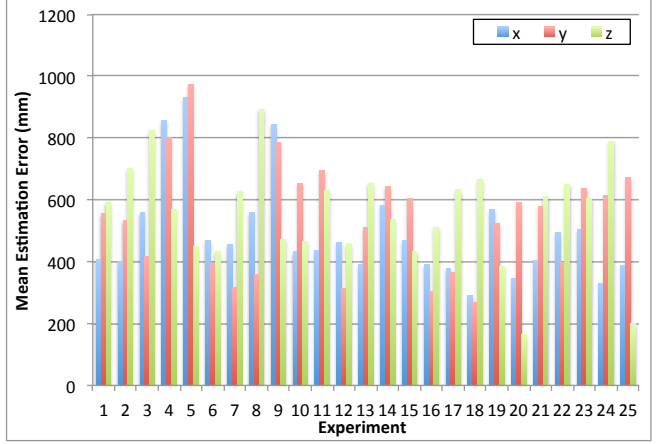

Fig. 12: Mean estimation error for each axis

to the actual position of the device. As shown in Fig. 12 the mean estimation error of the proposed localization technique is enough small for Smart Interaction System.

Besides, in case of the estimation error is larger than $1000 \mathrm{~mm}$, we expect that longer-term RSSI collection will reduce the estimation error from Fig. 9. Even if the estimation error is larger than $1000 \mathrm{~mm}$ and the icons are interchanged left and right on the display, the user can find the error of the icons once he operates the device from smartphone. The burden to modify positions of only few nodes is relatively smaller compared to one to input the position of all the nodes. Thus we consider the proposed localization technique and Smart Interaction System are practical to improve the operability of remote-control devices.

\section{CONCLUSIONS}

To improve operability of the remote-control devices, we proposed a 3D localization technique with a mobile robot. In the proposed localization technique, a mobile robot reduces the burden of deployment of anchors required in some existing techniques. The proposed localization technique takes into account the gain of omnidirectional antenna to estimate the position of the devices without the prior knowledge of channel condition. We have implemented the proposed localization technique and the Smart Interaction System with a mobile robot, sensors, a server and a smartphone. We conducted experiments with the implemented system to analyze the estimation error of the proposed localization technique. The experimental results show that the mean estimation error is approximately $1000 \mathrm{~mm}$. We consider that the proposed localization technique and the Smart Interaction System are practical for remotecontrol devices because the estimation error is enough small to allow a user to identify each device in a room with less burden. For the future work, we are performing an experiment in environment with the obstacles such as furniture.

\section{ACKNOWLEDGMENT}

We would like to thank Mr. Hanumantha Rao Madala for his support in Server programming part of the project.

\section{REFERENCES}

[1] N. Patwari, R. J. O'Dea, and Y. Wang. Relative Location in Wireless Networks. IEEE VTS 53rd Vehicular Technology Conference, vol. 2, pages 1149-1153, May 2001.

[2] G Zanca, F Zorzi, A Zanella, and M Zorzi. Experimental comparison of RSSI-based localization algorithms for indoor wireless sensor networks. In Proc. of the workshop on Realworld wireless sensor networks, pages 1-5, 2008.

[3] M. Sugano, T. Kawazoe, Y. Ohta, and M. Murata. Indoor Localization System using RSSI measurement of Wireless Sensor Network based on Zigbee standard. Wireless Sensor Networks, 2006.

[4] G. Wang and K. Yang. A new approach to sensor node localization using rss measurements in wireless sensor networks. IEEE TRANSACTION ON WIRELESS COMMUNICATIONS, vol. 10, no. 5, pages 1389-1395, May 2011.

[5] O.S. Oguejiofor, V.N. Okorogu, Adewale Abe, and B.O Osuesu. Outdoor localization system using rssi measurement of wireless sensor network. Int'l Journal of Innovative Technology and Exploring Engineering (IJITEE), vol. 2, pages 1-6, January 2013.

[6] S. Gansemer, U. GroBmann, and S. Hakobyan. Rssi-based euclidean distance algorithm for indoor positioning adapted for the use in dynamically changing wlan environments and multilevel buildings. In Proc. of Int'l Conference on Indoor positioning and Indoor Navigation(IPIN), pages 1-6, September 2010.

[7] A. Bose and C. H. Foh. A practical path loss model for indoor wifi positioning enhancement. In Proc. of 6th Int'l Conference on Information, Communication \& Signal Processing, pages 1-5, December 2007.

[8] Y. F. Solahuddin and R. Mardeni. Indoor empirical path loss prediction model for $2.4 \mathrm{ghz} 802.11 \mathrm{n}$ network. In Proc. of IEEE Int'l Conference on Control System, Computing and Engineering, pages 12-17, November 2011.

[9] N. A. Dieng, M. Charbit, C. Chaudet, L. Toutain, and T. B. Meriem. A multi-path data exclusion model for rssi- based indoor localization. In Proc. of 15th Int'l Symposium on Wireless Personal Multimedia Communications (WPMC), pages 336-340, September 2012.

[10] S. Samanta, Prof. Punesh, U.Tembhare, Prof. Charan, and R. Pote. A survey on 3d localization in wireless sensor networks. Int'l Journal Of Computational Engineering Research, vol. 3, no. 1, pages 90-94, January 2013.

[11] T. Chuenurajit, D. Suroso, and P. Cherntanomwong. Implementation of RSSI-Based 3D Indoor Localization using Wireless Sensor Networks Based on ZigBee Standard. Journal of information science and technology, vol. 3, issue 2, December 2012.

[12] J. Borenstein and L. Feng. Measurement and correction of systematic odometry errors in mobile robots. IEEE Transactions on Robotics and Automation, vol. 12, no. 6, pages 869-880, December 1996.

[13] iRobot. iRobot Roomba 500 Open Interface (OI) Specification. http://www.irobot.lv/uploaded_files/File/iRobot_ Roomba_500_Open_Interface_Spec.pdf.

[14] Indian Institute of Technology Hyderabad. 'iith mote' - wireless sensor communication module. http://www.iith.ac.in/ raji/downloads/IITH-mote-webpage.pdf. 